\title{
The Effect of Obesity on Operative Time and Postoperative Complications for Peritrochanteric Femur Fractures
} Hayden B. Schuette ${ }^{1}$, William M. Durkin ${ }^{2}$, Braden J. Passias ${ }^{1}$, Daniel DeGenova ${ }^{1}$, Carina Bertolini ${ }^{3}$,
Philip Myers ${ }^{4}$, Benjamin C. Taylor ${ }^{3}$

1. Orthopedic Surgery, OhioHealth, Columbus, USA 2. Orthopedics, Aultman Hospital, Canton, USA 3. Orthopedic Trauma, OhioHealth, Columbus, USA 4. Orthopedics, Singing River Hospital, Gulfport, USA

Corresponding author: Hayden B. Schuette, haydenschuette@gmail.com

\section{Abstract}

\section{Introduction}

The high prevalence of obesity among adults in the United States presents significant challenges to orthopedic surgeons. Obesity has been shown to increase operative time and complications in both elective and nonelective orthopedic surgeries. Despite this, there is a lack of literature evaluating the effect obesity has on operative time and postoperative complications following peritrochanteric fracture surgery.

\section{Methods and Materials}

We performed a retrospective review of patients who underwent isolated operative stabilization of a peritrochanteric femur fracture at our urban level one trauma center between 2010 and 2018. Patients were divided into an obese group, as defined by a body mass index (BMI) equal to or greater than $30 \mathrm{~kg} / \mathrm{m}^{2}$, and a nonobese group, as defined by a BMI less than $30 \mathrm{~kg} / \mathrm{m}^{2}$. Operative timing variables including time to surgery, operative time, total operating room (OR) time, anesthesia time, and fluoroscopy time were collected. Postoperative variables evaluated including the presence of a major postoperative complication within 90 days of surgery, the need for repeat surgery within 90 days, and the need for surgery due to an infection within 90 days were collected.

\section{Results}

A total of 175 patients were included in this retrospective review. Thirty-seven patients were included in the obese group, and 138 were included in the nonobese group. Obesity was associated with a significantly $(\mathrm{p}=$ $0.002)$ longer operative time, total OR time $(\mathrm{p}=0.0001)$, anesthesia time $(\mathrm{p}=0.00006)$, and fluoroscopy time $(p=0.0001)$. There was no significant difference $(p>0.05)$ in postoperative variables between the obese and nonobese group. The 90 -day major postoperative complication rate was $10.8 \%$ in the obese group and $10.9 \%$ in the nonobese group. Both repeat surgery and surgery for infection within 90 days were $2.7 \%$ in the obese group and $1.4 \%$ in the nonobese group.

Review began 10/25/2020 Review ended 11/13/2020 Published 11/26/2020

\section{(c) Copyright 2020}

Schuette et al. This is an open access article distributed under the terms of the Creative Commons Attribution License CC-BY 4.0., which permits unrestricted use, distribution, and reproduction in any medium, provided the original author and source are credited.

\section{Conclusion}

The treatment of peritrochanteric femur fractures in obese patients is associated with a significantly longer operative time, total OR time, anesthesia time, and fluoroscopy time, but no difference in major postoperative complications when compared to nonobese patients.

Categories: Orthopedics

Keywords: obesity, hip fracture, peritrochanteric fracture, operative time, postoperative complication

\section{Introduction}

Obesity presents significant healthcare challenges throughout all fields of medicine, including orthopedics. It is estimated that the prevalence of obesity among adults in the United States was 42.4\% in 2017-2018 [1]. Recent orthopedic literature has shown that obesity can lead to increased complications as well as longer operative times in both elective and nonelective orthopedic surgeries [2-6]. Like obesity, the aging population in the United States has also increased healthcare demand. Each year in the United States, over 300,000 people over the age of 65 are hospitalized for hip fractures [7]. Peritrochanteric fractures, defined as those occurring in the intertrochanteric or subtrochanteric region of the proximal femur, account for a large proportion of hip fractures. Treating peritrochanteric fractures in obese patients presents several potential challenges that must be considered by the patient as well as all providers involved in the patient's care.

A recent study found obesity to be associated with higher perioperative complication rates and longer operative times when treating intertrochanteric femur fractures [8]. In contrast, the concept of the "obesity 


\section{Cureus}

paradox" suggests that a high body mass index (BMI) is positively associated with survival following a hip fracture. The "obesity paradox" has been validated in several recent studies looking at post-hip fracture surgery morbidity and mortality $[9,10]$. Despite this, there remains a paucity of literature investigating the effect obesity has on operative time and postoperative complications following peritrochanteric fracture surgery.

The primary purpose of this study was to evaluate differences in operative times between obese and nonobese patients undergoing operative fixation of peritrochanteric femur fractures. Secondary goals of this study are to compare perioperative timing and postoperative complications within 90 days between obese and nonobese patients undergoing operative fixation of peritrochanteric femur fractures.

\section{Materials And Methods}

After formal institutional review board approval, we retrospectively reviewed the charts of 175 patients who underwent operative stabilization of a peritrochanteric femur fracture (Figure 1) at our urban level one trauma center between October 1, 2010 and October 31, 2018. Peritrochanteric fractures were defined as those occurring in the intertrochanteric or subtrochanteric region of the proximal femur. Prior to the initiation of this study, an a priori power analysis was performed in order to estimate sufficient sample size needed to achieve adequate power to identify differences in operative time.

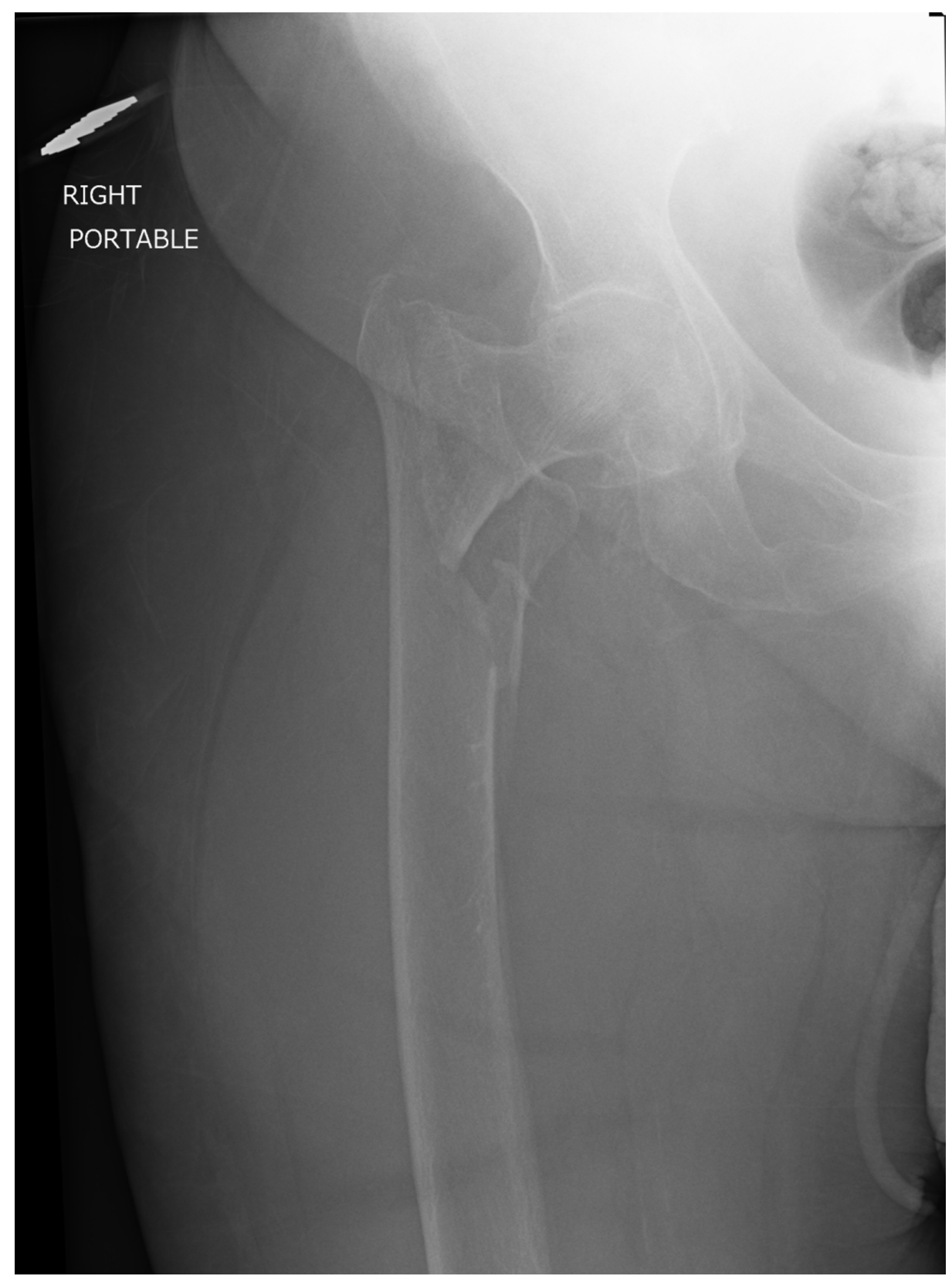

\section{FIGURE 1: Preoperative Radiograph}

An anteroposterior radiograph of the right hip demonstrating a displaced intertrochanteric femur fracture 


\section{Cureus}

The inclusion criteria were patient age equal to or greater than 18 years and patients undergoing an isolated operative procedure for a peritrochanteric femur fracture, including either cephalomedullary nailing (Figure 2) or open reduction and internal fixation (ORIF). Patients were excluded if they had less than three months of follow-up, had previous surgical treatment about the femur, or had multiple operative procedures performed under the same anesthesia episode.

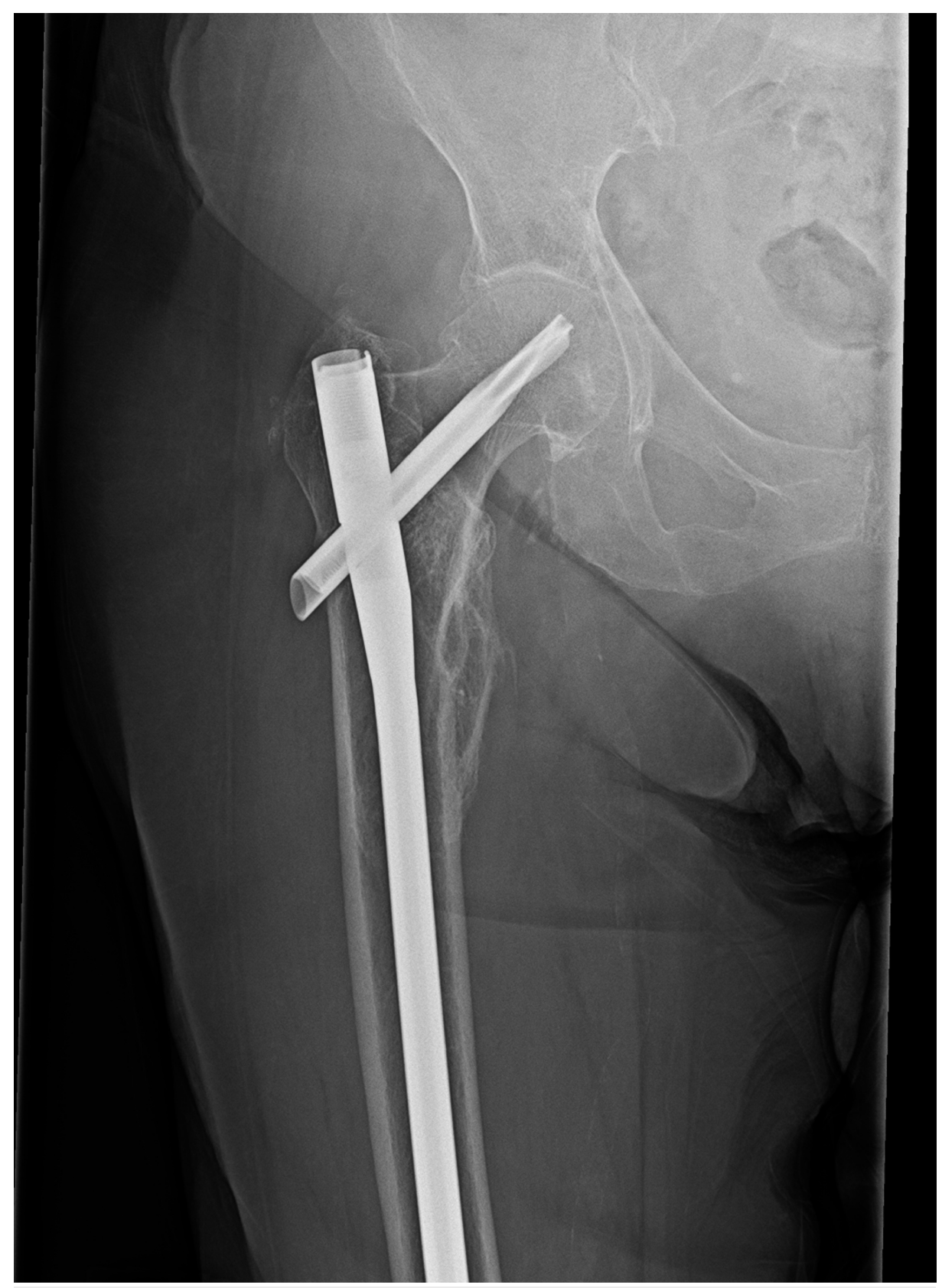

\section{FIGURE 2: Postoperative Radiograph}

An anteroposterior radiograph of the right hip one-year status post cephalomedullary nail for the fracture in Figure 1.

Patients were divided into an obese group, as defined by a BMI equal to or greater than $30 \mathrm{~kg} / \mathrm{m}^{2}$ and a nonobese group, as defined by a BMI less than $30 \mathrm{~kg} / \mathrm{m}^{2}$. Demographic data including age, sex, BMI, American Society of Anesthesiologists (ASA) Physical Status, presence of diabetes mellitus, and smoking status were collected. Operative variables collected included time to surgery, operative time, total operating 


\section{Cureus}

room (OR) time, anesthesia time, and fluoroscopy time. Postoperative variables including the presence of a major postoperative complication within 90 days of surgery, the need for repeat surgery within 90 days, and the need for surgery due to an infection within 90 days were collected. A major postoperative complication was defined as a myocardial infarction, stroke, deep vein thrombosis, pulmonary embolism, deep wound infection, or death. Additional surgeries within 90 days were defined as surgeries related to infection, hardware failure, or a new fracture about the previous operative site.

\section{Statistical analysis}

Statistical analysis was performed using Microsoft Excel (Redmond, WA). Data were collected and expressed as means, standard deviations, and percentages. Continuous variables were compared using student's t-test. Categorical variables were compared using chi-square test. An alpha-level of 0.05 was used to determine statistical significance.

\section{Results}

A total of 175 patients were included in this retrospective review (Table 1). Thirty-seven patients were included in the obese group, and 138 were included in the nonobese group. The obese group was comprised of significantly $(\mathrm{p}=0.02)$ more females $(72.9 \%$ versus $52 \%)$ and significantly $(\mathrm{p}=0.005)$ more diabetics (48.6\% versus $27.1 \%)$, while the nonobese group had significantly $(\mathrm{p}=0.007)$ more smokers ( $34 \%$ versus 13.5\%). The average BMI of the obese group was $35.6 \mathrm{~kg} / \mathrm{m}^{2}$, while the average BMI of the nonobese group was $23.3 \mathrm{~kg} / \mathrm{m}^{2}$.

\begin{tabular}{|c|c|c|c|}
\hline Variable & Obese Group & Nonobese Group & P Value \\
\hline Patients (number) & 37 & 138 & - \\
\hline Age (years) & $62 \pm 16.9$ & $67.5 \pm 20.8$ & 0.14 \\
\hline Sex (female) & $27(72.9 \%)$ & 72 (52\%) & 0.02 \\
\hline Body Mass Index $\left(\mathrm{kg} / \mathrm{m}^{2}\right)$ & $35.6 \pm 4.9$ & $23.3 \pm 3.4$ & $<0.00001$ \\
\hline ASA Physical Status & $3 \pm 0.64$ & $3.1 \pm 0.75$ & 0.43 \\
\hline Diabetic (yes) & $18(48.6 \%)$ & $34(27.1 \%)$ & 0.005 \\
\hline Smoker (yes) & $5(13.5 \%)$ & $51(34 \%)$ & 0.007 \\
\hline
\end{tabular}

\section{TABLE 1: Demographic Variables}

Noncategorical variables are expressed as mean \pm standard deviation. Categorical variables are given as absolute numbers with percentages in parentheses.

ASA, American Society of Anesthesiologists.

Operative variables are presented in Table 2 . There was no significant difference in time to surgery between the obese and nonobese groups. Obesity was associated with a significantly $(p=0.002)$ longer operative time, with the obese group having an average operative time of 73.2 minutes compared to 59.2 minutes in the nonobese group. Analysis of other operative variables demonstrates significantly longer total OR time ( $p$ $=0.0001)$, anesthesia time $(p=0.00006)$, and fluoroscopy time $(p=0.0001)$ in the obese group. 


\section{Cureus}

\begin{tabular}{|c|c|c|c|}
\hline Variable & Obese Group & Nonobese Group & P Value \\
\hline Time to surgery (hours) & $20.7 \pm 13.5$ & $24.4 \pm 28.1$ & 0.44 \\
\hline Operative time (minutes) & $73.2 \pm 25.4$ & $59.2 \pm 24.6$ & 0.002 \\
\hline Total operating room time (minutes) & $128.3 \pm 23.7$ & $110.6 \pm 24.4$ & 0.0001 \\
\hline Anesthesia time (minutes) & $133.1 \pm 24.5$ & $114.1 \pm 25.1$ & 0.00006 \\
\hline Fluoroscopy time (minutes) & $2.7 \pm 1.3$ & $1.9 \pm 1.0$ & 0.0001 \\
\hline
\end{tabular}

TABLE 2: Operative Variables

Variables are expressed as mean \pm standard deviation.

Table 3 presents the outcomes of postoperative variables. Between the obese and nonobese groups, there was no significant difference in major postoperative complications within 90 days, repeat surgery within 90 days, or surgery for an infection within 90 days. The 90 -day major postoperative complication rate was $10.8 \%$ in the obese group and $10.9 \%$ in the nonobese group. Both repeat surgery and surgery for infection within 90 days were $2.7 \%$ in the obese group and $1.4 \%$ in the nonobese group.

\begin{tabular}{|c|c|c|c|}
\hline Variable & Obese Group & Nonobese Group & P Value \\
\hline Major postoperative complication within 90 days (yes) & $4(10.8 \%)$ & $15(10.9 \%)$ & 0.99 \\
\hline Repeat surgery within 90 days (yes) & $1(2.7 \%)$ & $2(1.4 \%)$ & 0.6 \\
\hline Surgery for infection within 90 days (yes) & $1(2.7 \%)$ & $2(1.4 \%)$ & 0.6 \\
\hline
\end{tabular}

\section{TABLE 3: Postoperative Variables}

Categorical variables are given as absolute numbers with percentages in parentheses.

\section{Discussion}

This study is one of the few reported series comparing perioperative timing as well as postoperative complications between obese and nonobese patients who underwent operative stabilization of peritrochanteric femur fractures. We found obesity to be associated with a significantly longer operative time; however, there was no difference in major postoperative complications, repeat surgery, or surgery for an infection within 90 days postoperatively when comparing obese to nonobese patients undergoing operative fixation of peritrochanteric fractures. Additionally, we found the obese patients to have significantly longer total OR time, anesthesia time, and fluoroscopy time.

In our study, operative time, total OR time, anesthesia time, and fluoroscopy time were found to be significantly longer in the obese group than in the nonobese group. Previous studies have found obesity to be associated with longer operative times when treating hip fractures $[8,11]$. In a multicenter retrospective study investigating the effect of obesity on perioperative complications in those undergoing surgical treatment of intertrochanteric femur fractures, Kempegowda et al. found operative time to be statistically longer in obese (96 minutes) versus nonobese (86 minutes) patients [8]. Similarly, Akinleye et al. evaluated patients undergoing a variety of operative treatments for hip fractures and found mean operative time to have a linear relationship with increasing BMI [11]. It is likely that this increased operative time, as well as total OR time found in our study, is due to a multitude of factors. These factors likely include increased difficulty with patient positioning and intraoperative imaging as well as increased difficulty with fracture reduction that may necessitate more frequent need for open reduction. Our study and others demonstrate that operative treatment of hip fractures in obese patients takes significantly more time and work than nonobese patients.

The American Medical Association's Current Procedural Terminology (CPT) system standardizes healthcare services and procedures and assigns value to them based on the amount of physician time, skill, work, and risk involved. The 22-modifier allows for increased reimbursement in cases when a procedure requires work that is considered more challenging than typical for the procedure being performed [12]. It has recently been reported that the use of the 22-modifier in total hip arthroplasty resulted in significantly higher 
reimbursement when used in obese patients [13]. Based on our findings, we believe that the use of the 22modifier for peritrochanteric fracture surgery in obese patients is appropriate. However, further studies evaluating the financial implications of the 22-modifier for peritrochanteric hip fracture surgery is needed, as our study does not address this.

We found no significant difference between obese and nonobese patients in postoperative complications, infection, or need for repeat surgery within 90 days. In a study looking at postoperative medical complications following hip fracture surgery, Batsis et al. found that BMI had no significant influence on postoperative non-cardiac medical complications [14]. In contrast, Kepegowda et al. found obesity to be significantly associated with increased overall postoperative complications $(43 \%$ vs. $28 \%, \mathrm{p}<0.0001)$ in the treatment of intertrochanteric femur fractures [8]. Unlike in total joint arthroplasty, where obesity has been clearly defined as a risk factor for postoperative complications, it appears the relationship between BMI and postoperative complications for hip fracture surgery remains ill-defined [15-17].

As with any study, several inherent weaknesses are present. First, the follow-up period in this study is only 90 days. It is possible that not all major postoperative outcomes would occur in this 90-day period, and therefore our study may underestimate the true postoperative complication rates. Next, the effect of the specific surgical procedure was not evaluated in this study; it is possible that outcomes may be altered if patients undergoing only intramedullary nailing or only ORIF were evaluated. Lastly, the retrospective nature of this study carries an inherent bias that would be improved with prospective data collection.

\section{Conclusions}

Our study shows that the operative treatment of peritrochanteric femur fractures in obese patients is associated with a significantly longer operative time, total OR time, anesthesia time, and fluoroscopy time. However, our study does not demonstrate a significant difference in major postoperative complications when comparing obese and nonobese patients undergoing operative treatment of peritrochanteric femur fractures. Orthopedic surgeons should plan for increased operative time for obese patients with peritrochanteric femur fractures. Lastly, further investigations are necessary to evaluate the effect obesity has on postoperative complications following operative treatment of peritrochanteric femur fractures.

\section{Additional Information \\ Disclosures}

Human subjects: Consent was obtained by all participants in this study. OhioHealth Corporation Institutional Review Board issued approval 1367251. Animal subjects: All authors have confirmed that this study did not involve animal subjects or tissue. Conflicts of interest: In compliance with the ICMJE uniform disclosure form, all authors declare the following: Payment/services info: All authors have declared that no financial support was received from any organization for the submitted work. Financial relationships: Benjamin C. Taylor, MD declare(s) personal fees and royalties from Zimmer Biomet. Benjamin C. Taylor, MD declare(s) royalties from Innomed. Benjamin C. Taylor, MD declare(s) Editorial board member from Orthobullets. Other relationships: All authors have declared that there are no other relationships or activities that could appear to have influenced the submitted work.

\section{References}

1. Prevalence of obesity and severe obesity among adults: United States, 2017-2018 . (2020). Accessed: September 4, 2020: https://www.cdc.gov/nchs/products/databriefs/db360.htm.

2. Childs BR, Nahm NJ, Dolenc AJ, Vallier HA: Obesity is associated with more complications and longer hospital stays after orthopaedic trauma. J Orthop Trauma. 2015, 29:504-509. 10.1097/BOT.0000000000000324

3. Liabaud B, Patrick DA, Jr., Geller JA: Higher body mass index leads to longer operative time in total knee arthroplasty. J Arthroplasty. 2013, 28:563-565. 10.1016/j.arth.2012.07.037

4. Raphael IJ, Parmar M, Mehrganpour N, Sharkey PF, Parvizi J: Obesity and operative time in primary total joint arthroplasty. J Knee Surg. 2013, 26:95-99. 10.1055/s-0033-1333663

5. Tucker MC, Schwappach JR, Leighton RK, Coupe K, Ricci WM: Results of femoral intramedullary nailing in patients who are obese versus those who are not obese: a prospective multicenter comparison study. J Orthop Trauma. 2007, 21:523-529. 10.1097/BOT.0b013e31813347ac

6. Weinlein JC, Deaderick S, Murphy RF: Morbid obesity increases the risk for systemic complications in patients with femoral shaft fractures. J Orthop Trauma. 2015, 29:91-95. 10.1097/BOT.0000000000000167

7. Hip fractures among older adults. (2016). Accessed: September 4, 2020: https://www.cdc.gov/homeandrecreationalsafety/falls/adulthipfx.html.

8. Kempegowda H, Richard R, Borade A, et al.: Obesity is associated with high perioperative complications among surgically treated intertrochanteric fracture of the femur. J Orthop Trauma. 2017, 31:352-357. 10.1097/BOT.0000000000000825

9. Modig K, Erdefelt A, Mellner C, Cederholm T, Talbäck, Hedström M: “Obesity paradox” holds true for patients with hip fracture: a registry-based cohort study. J Bone Joint Surg Am. 2019, 101:888-895. 10.2106/JBJS.18.01249

10. Flodin L, Laurin A, Lökk J, Cederholm T, Hedström M: Increased 1-year survival and discharge to independent living in overweight hip fracture patients: a prospective study of 843 patients. Acta Orthop. 


\section{Cureus}

2016, 87:146-151. 10.3109/17453674.2015.1125282

11. Akinleye SD, Garofolo G, Culbertson MD, Homel P, Erez O: The role of BMI in hip fracture surgery . Geriatr Orthop Surg Rehabil. 2018, 9:2151458517747414. 10.1177/2151458517747414

12. Filler BC: Coding basics for orthopaedic surgeons. Clin Orthop Relat Res. 2007, 457:105-113.

10.1097/blo.0b013e31803372b8

13. Smith EL, Tybor DJ, Daniell HD, Naccarato LA, Pevear ME, Cassidy C: The 22-modifier in reimbursement for orthopedic procedures: hip arthroplasty and obesity are worth the effort. J Arthroplasty. 2018, 33:20472049. 10.1016/j.arth.2018.02.058

14. Batsis JA, Huddleston J, Melton 3rd LJ, Huddleston PM, Larson DR, Gullerud RE, McMahon MM: Body mass index and risk of non-cardiac post-operative medical complications in elderly hip fracture patients: a population-based study. J Hosp Med. 2009, 4:E1-9. 10.1002/jhm.527

15. DeMik DE, Bedard NA, Dowdle SB, Elkins JM, Brown TS, Gao T, Callaghan JJ: Complications and obesity in arthroplasty- a hip is not a knee. J Arthroplasty. 2018, 33:3281-3287. 10.1016/j.arth.2018.02.073

16. Jeschke E, Citak M, Günster C, et al.: Obesity increases the risk of postoperative complications and revision rates following primary total hip arthroplasty: an analysis of 131,576 total hip arthroplasty cases. J Arthroplasty. 2018, 33:2287-2292. 10.1016/j.arth.2018.02.036

17. Zusmanovich M, Kester BS, Schwarzkopf R: Postoperative complications of total joint arthroplasty in obese patients stratified by BMI. J Arthroplasty. 2018, 33:856-864. 10.1016/j.arth.2017.09.067 